Result. We identified a wide range of flaws with the current system and implementation of monitoring, and difficulty in locating the required information. There was no consistent monitoring of physical observations on electronic record, nor an accepted alternative way in which this was documented. Furthermore, blood tests were not consistently obtained either by the service or GP practices in a reproducible manner. This led to discussions within the CMHT regarding creation of a new pathway for the monitoring of this patient cohort using a Quality Improvement model, with the ultimate goal to establish a regular physical health clinic.

Conclusion. There is significant evidence that patients with major mental disorder do not access healthcare as consistently as those without, leading to a disparity in life expectancy. In light of the fact that antipsychotic medications can be associated with Metabolic Syndrome, we have an even greater responsibility to tackle this marked health inequality by appropriately monitoring our patients. This was not done well in this particular CMHT, but this project will lead to improvements in the service and ultimately patient care.

Does the advice requested by carers of people who live with dementia reflect the level of commissioned post-diagnostic support? A retrospective evaluation of calls to the Me2U dementia day centre 24-hour advice line

Rajan Nathan ${ }^{1 \star}$, Stephen Callaghan ${ }^{2}$, Kelly Walker ${ }^{3}$, Angela Mason $^{3}$ and Rosemarie Whittington ${ }^{3}$

${ }^{1}$ Cheshire and Wirral Partnership NHS Foundation Trust; ${ }^{2} \mathrm{EQE}$ Health Ltd and ${ }^{3} \mathrm{Me} 2 \mathrm{u}$ Dementia Day Centre

${ }^{\star}$ Corresponding author.

doi: 10.1192/bjo.2021.884

Aims. The aim was to examine the reasons for advice requests by carers of people who live with dementia (PLWD) that attend the $\mathrm{Me} 2 \mathrm{u}$ dementia day centre in order to identify key explanatory themes. We hypothesised that requests were related mainly to coordinating care and clinical issues due to limited postdiagnostic support (PDS) in our area.

Background. The Me2u dementia day centre (Merseyside) cares for PLWD and also supports carers. As part of the service, a 24-hour advice line is included for PLWD and their carers who attend the centre. Locally, there is limited PDS and most carers navigate the health and social care system alone mirroring the findings by the National Collaborating Centre for Mental Health (NCCMH).

Method. We undertook a retrospective evaluation of 244 advice calls, from 64 carers, between 01/06/2019 and 31/12/2019. We analysed time of call, type of advice, type of dementia, age and whether the advice was for the PLWD or for the carer.

Result. Of the 244 calls, the most common time to call was between $09.00-14.00(\mathrm{n}=168$; $(68.8 \%)$ peak $09.00-10.00(\mathrm{n}=$ 38). Average age of the person about whom the advice was sought was 79.08 years. $91.4 \%$ of the advice calls related to PLWD (most common dementia Alzheimer's) and $8.6 \%$ to the carer only. The mean number of calls per person was 3.8 (range 1-24).

Advice data were grouped into 9 broad themes namely, related to symptoms/behaviour $(32.79 \%, \mathrm{n}=80)$, request for $\mathrm{Me} 2 \mathrm{u}$ to coordinate care $(20.08 \%, \mathrm{n}=49)$, general advice $(14.75 \%, \mathrm{n}=36)$, personal care $(9.42 \%, \mathrm{n}=23)$, carer only advice $(8.60 \%, \mathrm{n}=21)$, social issues $(6.14 \%, \mathrm{n}=15)$, social care $(4.50 \%, \mathrm{n}=11)$, safeguarding $(2.46 \%, n=6)$, non-health and social care issue $(1.23 \%, n=3)$. Conclusion. Reasons for limited/poor PDS given by the NCCMH are; absence of named coordinators of care, over-reliance on families and carers to manage and facilitate appointments, poor recognition and management of comorbidities. This data show that $52.87 \%$ of calls were for clinical advice and coordination of care reflecting NCCMH findings. The interventions post-call reduced the impact on providers of urgent care.

These findings provide support for the provision of a [24-hour] advice line as a routine part of post-diagnostic support services, especially in areas that have limited or poor PDS. Commissioners of PDS services in areas that have limited or poor PDS should make this a priority to prevent unplanned admissions to hospital and carer breakdown.

\section{Vitamin D monitoring and management within men's} secure services

Jason Niblett ${ }^{1 \star}$, Shay-Anne Pantall ${ }^{2}$ and Anis Ahmed ${ }^{2}$

${ }^{1}$ University of Birmingham, Medical School and ${ }^{2}$ Birmingham and Solihull Mental Health NHS Foundation Trust

${ }^{*}$ Corresponding author.

doi: 10.1192/bjo.2021.885

Aims. To audit the investigation, identification and treatment of Vitamin D deficiency within Men's Secure Services.

Background. Vitamin D and/or vitamin D deficiency has been suggested to play a role in the pathogenesis of mental illness. There is evidence that Vitamin D inadequacy is pandemic among rehabilitation patients in inpatient settings. Patients within secure hospitals are similarly considered to be at high risk, due to their limited solar exposure during often lengthy admissions. It has been suggested that these patients should be considered an 'at-risk' cohort, for whom Vitamin D supplementation should be routine. Men's secure services in Birmingham comprise of two medium secure units and a low secure rehabilitation unit. Here we present an audit of Vitamin D monitoring and treatment completed in 2019. Method. A three year retrospective review of electronic patient records, for all inpatients admitted within men's secure services as of 1 September $2019(n=188)$. Standards were based on the Trust accepted guidelines for management of Vitamin D deficiency. Result. Key findings included:-

The majority of inpatients were Caucasian (43\%) and AfricanCaribbean (24\%). Ages ranged from 18 to 70 , with a mean age of 39. Approximately two-thirds (65\%) had been in hospital for over a year, of which $44 \%$ had been admitted for more than 3 years. The average length of admission was 885 days.

Only $47 \%$ of patients had their Vitamin D level checked within the study period.

Of those checked, $24 \%$ were tested within 1 month of admission. The mean duration between admission and Vitamin D testing was 464 days.

Results ranged from 10.3 to $118.5 \mathrm{nmol} / \mathrm{L}$. A high rate of Vitamin D deficiency was identified (54\%), whilst a further $16 \%$ had 'inadequate' levels.

$23 \%$ of those identified as requiring treatment did not receive any supplementation, whilst $59 \%$ of those with sufficient Vitamin D were prescribed treatment.

Only $48 \%$ had their levels rechecked following treatment; of these, only $59 \%$ now had an adequate Vitamin D status.

Conclusion. This audit demonstrates limited Vitamin D monitoring within male forensic inpatients. There was a high prevalence of Vitamin D insufficiency in this population, yet a substantial proportion of patients with identified deficiency were not prescribed any treatment. Ongoing monitoring and review of 
treatment effectiveness was poor. We argue that more consideration should be given to this population, with robust guidelines introduced for the treatment of this specific 'at-risk group'.

An audit looking at the impact of poverty on referrals to child and adolescent mental health services

Aida Nourbakhsh ${ }^{1 *}$, Kandarp Joshi ${ }^{2}$ and Breige Yorston ${ }^{3}$

${ }^{1}$ University of Aberdeen; ${ }^{2}$ Child and Adolescent Mental Health

Services NHS Grampian, University of Aberdeen and ${ }^{3}$ CAMHS NHS Grampian

${ }^{*}$ Corresponding author.

doi: 10.1192/bjo.2021.886

Aims. Recently, there has been a greater focus on how mental health in young people (YP) can be improved. Up to $10 \%$ of YP in Scotland have a diagnosable mental health condition1 and half of all adults with mental ill-health have had symptoms from their mid-teens2. Poverty is an important factor associated with poorer mental well-being from an early age which worsens if left untreated3. The aim of this audit was to answer the question: Are more YP referred from the least deprived areas, and are they more likely to require medication intervention or high intensity (tier 4) care? The results of which could help identify possible avenues for intervention to help improve retention of those most at risk of negative outcomes.

Method. NHS Grampian CAMHS provides service to Aberdeen City, Aberdeenshire, and Moray. Pre-collected data over 15 months from these areas were analysed using the Scottish Index of Multiple Deprivation (SIMD) deciles to distinguish any differences between referrals made. In addition, this audit evaluated the data to define any trends of deprivation linking YP to medication intervention or tier 4 care.

Result. Results showed that more referrals were made for YP in low-ranking areas $(3.19 \%$ of decile one compared to $1.74 \%$ of decile ten). The referrals were also more likely to be rejected based on the referral criteria, $33 \%$ in decile one versus $21 \%$ in decile ten. The increased rejection of referrals is most likely a reflection of the health inequalities faced by communities in more deprived areas. In terms of service provision, the patients from the most deprived areas are 3 times more like to require tier 4 care while the least deprived are 1.5 times more likely as compared to percentage of population. With regards to medication intervention patients from deciles one, five, six and seven have significantly higher numbers.

Conclusion. This project set out to look at the current service provided by CAMHS and found that despite best efforts deprivation has had an impact on the acceptance of referrals. Going forward this data will be shared with multiagency stakeholders to develop service provisions, in particular the issues identified with the rejection of referrals in more deprived areas. Higher level of medication use in more deprived population is not unexpected but highlights the need to share the findings with a multiagency network.

\section{Evaluation of an attention deficit hyperactivity} disorder (ADHD) assessment \& treatment service

Chan Nyein ${ }^{1 \star}$ and David Oyewole ${ }^{2}$

${ }^{1}$ Northwick Park Mental Health Unit and ${ }^{2}$ Northwick Park Mental Health Unit, CNWL Adult ADHD Clinic

${ }^{*}$ Corresponding author.

doi: 10.1192/bjo.2021.887
Aims. The Central and North West London NHS Foundation Trust ADHD clinic offers diagnosis and medication stabilisation for adults with ADHD, in preparation for discharge back to GP for continued prescribing and monitoring. Referral waiting time is shortened by efficiently managing the service and soon transfer of care to GP whilst referrals have been increasingly accepted years on years. A snap shot service evaluation was made to understand characteristics of service exploring its strength and areas to improve.

Method. All 115 patients offered in March and April 2019 for an ADHD specialist assessment were sampled from the new electronic patient record SystmOne in use since 1st March 2019.

Data were collected for

Male \& Female ratio

Age range distribution

Clinical Commissioning Group referral source

Clinic attendance characteristics

ADHD diagnosis, sub-types and psychiatric comorbidity

ADHD Medication prescribed

FP10 Prescription duration by prescribers

Patient data were anonymously encoded into Microsoft Excel Sheet for sorting, counting, summating and illustrating into tables and pie charts.

Result. The male \& female ratio of the sample was $6: 5$ and nearly half were in age range 20-29 years. Majority were referred from Westminster and West London Clinical Commissioning Groups.

107 patients completed the assessment, of which 106 were diagnosed as having an adult ADHD.

$22 \%$ of follow-up clinics were cancelled or not attended (DNA) by patients. The majority of the patients (62\%) required $1-2$ follow-ups before transfer to GP, whilst $8 \%$ did not require or want follow-ups either already being on ADHD medication, not wanting medication or having lost to reviews. Only 3\% require six or more follow-ups.

Majority were reviewed after two- to five-week prescription, the peak being four-weekly.

91\% of completion to GP were discharged on ADHD medication, majority being singly on Elvanse (48\%) and Concerta XL (25\%). Discharge without ADHD medication was due to concerns for its addiction, preference on non-medication treatment, intolerance of medication adverse effect or mental health priority treatment.

Conclusion. Collaboration with GPs for their pre-treatment physical health screening facilitated prompt prescribing initiation on assessment with most discharges taken place after 1-2 follow-ups, enabling service turn-over with short waiting time (6-9 months in 2018/2019). Service expansion for increasing referral uptake is probably feasible from this baseline by appointing additional sessional clinicians and further efficiency management on clinic scheduling \& DNA with a target majority likely requiring 1-2 follow-ups with average four-weekly prescribing.

\section{Results of a client satisfaction questionnaire in a NHS psychotherapy department}

Elizabeth Ogston*, Kim Herbert and Lorraine McGuiness

NHS Lanarkshire

${ }^{*}$ Corresponding author.

doi: $10.1192 /$ bjo.2021.888

Aims. This study aimed to assess the level of satisfaction patients feel towards their experience of attending for psychotherapy, in order to inform local management on the service being offered by the department. 